\title{
Analysis of sentinel lymph node biopsy and non-sentinel lymph node metastasis in invasive ductal and invasive lobular breast cancer: a nationwide cross-sectional study (CSBrS-001)
}

\author{
Juliang Zhang ${ }^{1 \#}$, Yuwei Ling ${ }^{2 \#}$, Ting Wang ${ }^{1}$, Changjiao Yan ${ }^{1}$, Meiling Huang ${ }^{1}$, Zhimin Fan ${ }^{3}$, Rui Ling ${ }^{1}$; \\ Chinese Society of Breast Surgery
}

${ }^{1}$ Department of Thyroid, Breast and Vascular Surgery, Xijing Hospital, The Fourth Military Medical University, Xi'an, China; ${ }^{2}$ Center for Thyroid and Breast Surgery, Department of General Surgery, Xuanwu Hospital, Capital Medical University, Beijing, China; ${ }^{3}$ Department of Breast Surgery, The First Affiliated Hospital of Jilin University, Changchun, China

Contributions: (I) Conception and design: R Ling, Z Fan, J Zhang; (II) Administrative support: R Ling, Z Fan; (III) Provision of study materials or patients: Chinese Society of Breast Surgery; (IV) Collection and assembly of data: Chinese Society of Breast Surgery; (V) Data analysis and interpretation: Y Ling, C Yan; (VI) Manuscript writing: All authors; (VII) Final approval of manuscript: All authors.

\#These authors contributed equally to this work.

Correspondence to: Rui Ling. Department of Thyroid, Breast and Vascular Surgery, Xijing Hospital, The Fourth Military Medical University, 127 West Chang-Le Road, Xi'an 710032, China. Email: lingruiaoxue@126.com; Zhimin Fan. Department of Breast Surgery, The First Affiliated Hospital of Jilin University, 71 Xinmin Street, Changchun 130021, China. Email: fanzm@jlu.edu.cn.

Background: Information regarding the implementation of sentinel lymph node biopsy (SLNB) in invasive lobular carcinoma (ILC) is scarce, and whether ILC patients with 1-2 positive sentinel lymph nodes (SLNs) can be omitted from axillary lymph node dissection (ALND) remains controversial. This study aimed to compare involvement of SLNs and non-SLNs between patients with invasive ductal carcinoma (IDC) and ILC.

Methods: We retrospectively collected the clinical and pathological data of invasive breast cancer patients from 37 medical centers in China from January 2018 to December 2018. The number of resected SLNs, positive rate of SLNs, and non-SLNs metastasis were compared between patients with IDC and ILC.

Results: A total of 6,922 patients were included, comprising 6,650 with IDC (96.1\%) and 272 with ILC (3.9\%). No difference was observed in the number of resected SLNs between patients with IDC and ILC (IDC: $4.0 \pm 1.9 v s$. ILC: $3.9 \pm 1.6, \mathrm{P}=0.352$ ). The positive rate of SLNs was significantly higher in patients with IDC than that in patients with ILC (19.3\% in IDC vs. $12.9 \%$ in ILC, $\mathrm{P}=0.008)$. The difference in positive rate of SLNs between IDC and ILC was mainly attributed to macro-metastasis. For patients with positive SLNs who received ALND, and those with 1-2 positive SLNs, the metastatic rate of non-SLNs in the ILC group was higher than that in the IDC group (for patients with positive SLNs: $50.0 \%$ in ILC vs. $39.9 \%$ in IDC, $\mathrm{P}=0.317$; for patients with $1-2$ positive SLNs: $45.4 \%$ in ILC vs. $34.8 \%$ in IDC, $\mathrm{P}=0.366$ ), but the differences were not statistically significant.

Conclusions: Patients with ILC had similar number of resected SLNs and lower positive rate of SLNs compared to those with IDC. In participants with 1-2 positive SLNs, the ILC group had an increased tendency for non-SLNs metastasis compared with the IDC group. Surgeons may need to be more cautious about omitting ALND for ILC patients with 1-2 positive SLNs.

Keywords: Breast cancer; invasive lobular carcinoma (ILC); sentinel lymph node biopsy (SLNB); non-sentinel lymph node metastasis

Submitted Sep 02, 2021. Accepted for publication Oct 20, 2021.

doi: $10.21037 /$ atm-21-5169

View this article at: https://dx.doi.org/10.21037/atm-21-5169 


\section{Introduction}

Axillary lymph node status is a major prognostic factor in early-stage breast cancer (1). Axillary lymph node dissection (ALND) has been regarded the most accurate method for assessing metastatic spread of lymph nodes. However, ALND may result in lymphedema, motor deficit, and dysesthesia of the operated arm (2). Sentinel lymph node biopsy (SLNB) has spared the morbidity of ALND without compromising diagnostic accuracy and prognostic information (3). Sentinel lymph node metastasis $>2 \mathrm{~mm}$, the number of examined sentinel lymph nodes (SLNs) and proportion of involved SLNs have been identified correlated with axillary lymph node metastasis (4). In addition, the multifocality of primary tumor, tumor size, and lymph vascular invasion have been shown to be independent predictors of axillary lymph node metastasis (5). Progesterone receptor status correlated with axillary lymph node involvement, but in other studies the estrogen, progesterone or HER-2 receptor status has not been found to be consistently related to lymph node status (6).

In primary breast cancer, SLNB has supplanted ALND and been established as the standard surgical procedure for staging clinically negative nodes $(7,8)$. The American College of Surgical Oncology Group Z0011 (ACOSOG Z0011) study demonstrated that the omission of ALND among cT1-2N0M0 patients with 1-2 positive SLNs did not result in an inferior outcome compared with patients who underwent ALND (9). After the ACOSOG Z0011 clinical trial was revealed, the use of SLNB increased (10). However, most cases included in previous studies on SLNB were invasive ductal carcinoma (IDC). Information regarding the implementation of SLNB in patients with invasive lobular carcinoma (ILC) is sparse. Whether ILC patients with 1-2 positive SLNs can be exempted from ALND remains controversial (11-13).

Although ILC only accounts for approximately $5-10 \%$ of all breast cancer cases, the clinical course of ILC has unique aspects and merits special attention (14). The typical pathological characteristics of ILC are hormone receptor expression and absence of immunohistochemical (IHC) staining for E-cadherin (15). The peculiar growth pattern of ILC makes it difficult to diagnose clinically, as it usually lacks well-defined margins and does not form a palpable lesion (16); however, it tends to be hormone receptor positive and human epidermal growth factor receptor 2 (HER2) negative (14). Compared to IDC, the response of ILC to chemotherapy is significantly lower (17). It remains controversial whether IDC or ILC has a higher risk of
non-SLNs involvement, and there is little information regarding the implementation of SLNB in patients with ILC. In our previous study, we described implementation status of SLNB and subsequent processing of positive SLNs among breast cancer patients in China (18). To explore the aforementioned issues, this nationwide cross-sectional study compared the involvement of SLNs and non-SLNs between patients with IDC and ILC. We present the following article in accordance with the STROBE reporting checklist (available at https://dx.doi.org/10.21037/atm-21-5169).

\section{Methods}

\section{Patients}

In this nationwide cross-sectional study, we retrospectively collected clinical and pathological data of breast cancer patients from 37 medical centers in China based on the assistance of Chinese Society of Breast Surgery (CSBrS) (Table 1). A total of 12,233 breast cancer patients were admitted to these medical centers and received SLNB surgery from January 2018 to December 2018. The number of cases in the area during the study period determined the sample size. The inclusion criteria were as follows: (I) invasive ductal or invasive lobular breast cancer confirmed by postoperative pathology, (II) underwent SLNB. The exclusion criteria were as follows: (I) male, (II) carcinoma in situ without invasive disease, or histologic subtypes other than IDC or ILC, including mixed-type lobular cancer, (III) with metastatic disease at diagnosis, (IV) without complete medical record. Finally, 6,650 patients with IDC and 272 patients with ILC were enrolled in this study. The study flow chart is shown in Figure 1. We retrospectively collected detailed SLNB and clinicopathologic data from patient records. This study conformed to the provisions of the Declaration of Helsinki (as revised in 2013) and was approved by the Ethical Committee of Xijing Hospital, The Fourth Military Medical University (KY20192114-C-1). Individual consent for this retrospective analysis was waived.

\section{SLN evaluation}

The SLNB technique and classification of molecular subtype were performed as described in our previous study (18). The CSBrS issued a unified data collection form to 37 medical centers in China and defined the classification criteria in the collection form. Uniform data collection methods and classification standards reduced the information bias of this study. The SLNs 
Table 1 Distribution of enrolled invasive breast cancer patients in China

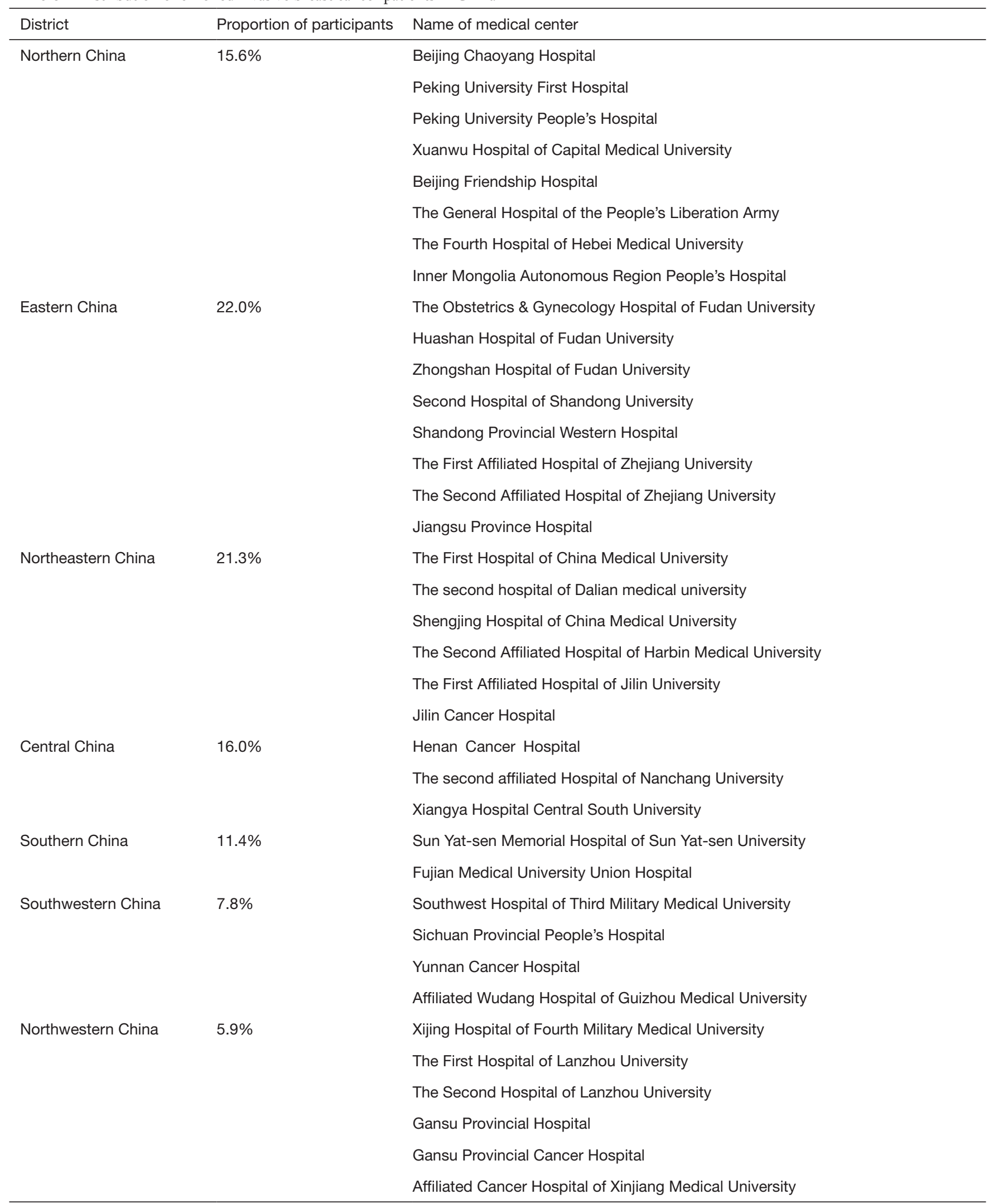




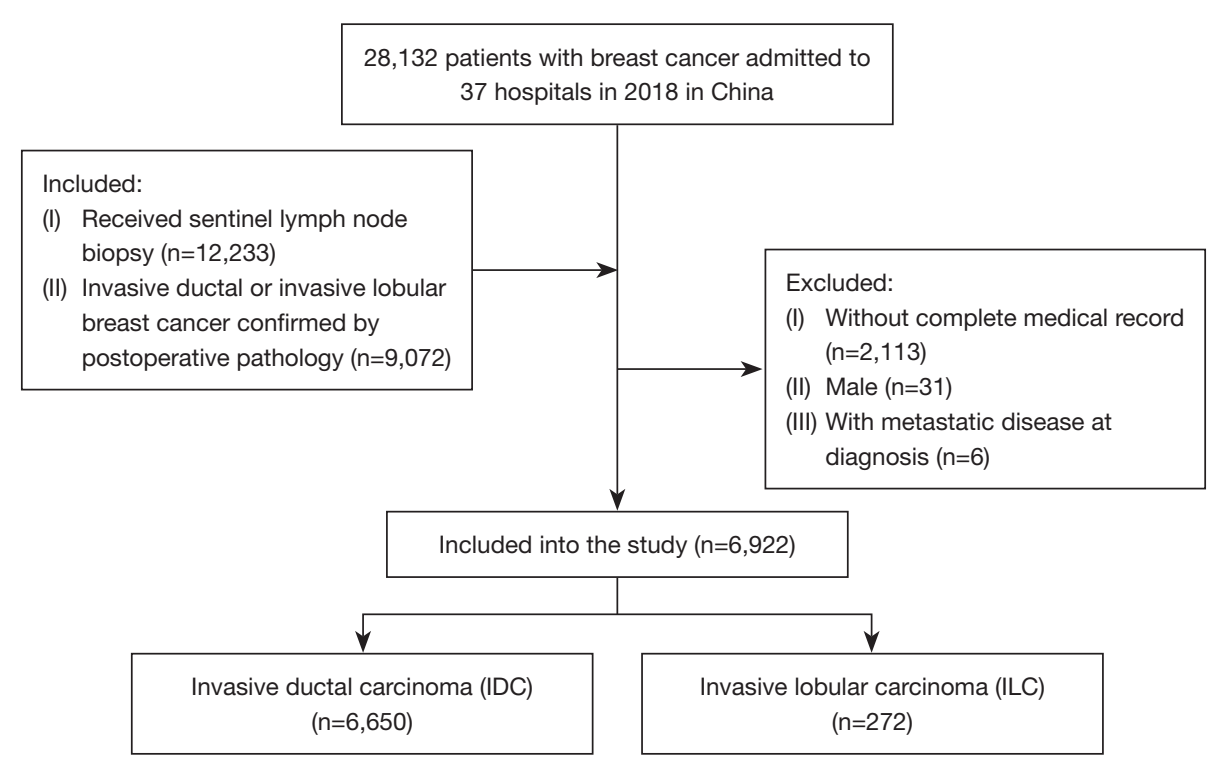

Figure 1 Study flow chart.

were considered positive for metastasis if they contained a macro-metastasis (deposit $>2 \mathrm{~mm}$ ) or a micro-metastasis (deposit $\geq 0.2$ to $2 \mathrm{~mm}$ ). The positive rate comprised the number of participants with metastatic SLNs/the number of participants who underwent SLNB $\times 100 \%$. Sentinel lymph node ratio (SLNR) was the number of positive SLNs/the total number of removed SLNs.

\section{Statistical analysis}

All statistical analyses were performed using the software SPSS version 23.0 (IBM Corp., Armonk, NY, USA). Normally distributed data were represented by mean \pm standard deviation. Cases with missing data were excluded. Student's $t$-test and univariate analysis of variance (ANOVA) were used to compare the difference of continuous variables. Categorical data was compared using $\chi^{2}$ test. For the multivariable analyses, binary logistic regression was used. Odds ratios (ORs) with their corresponding $95 \%$ confidence intervals ( $95 \%$ CIs) were calculated in multivariable analyses. All $\mathrm{P}$ values were 2-tailed, and $\mathrm{P}<0.05$ was considered statistically significant.

\section{Results}

\section{Participants and tumor characteristics}

Participant characteristics and pathological tumor characteristics are shown in Table 2. The study group consisted of 6,650 patients with IDC (96.1\%) and 272 patients with ILC (3.9\%). The main reason for excluding patients with metastatic disease at diagnosis and male patients is that these patients account for a very small proportion, and this group of people may show different SLNs and non-SLNs involvement. Several significant differences between the IDC participant group and ILC participants group were observed, including age at diagnosis, molecular subtype, and pathologic tumor size. Participants with ILC were older at diagnosis than those with IDC $(9.2 \% \leq 40$ years old in ILC $v s .14 .8 \%$ $\leq 40$ years old in IDC, $29.0 \%>60$ years old in ILC vs. $22.3 \%$ $>60$ years old in IDC, $\mathrm{P}=0.004)$. The participants with ILC were more associated with luminal $\mathrm{A}$ and less associated with HER2 enriched or triple-negative (TN) than those with IDC (41.9\% with luminal A in ILC vs. $29.6 \%$ with luminal A in IDC, $4.0 \%$ with HER2 enriched in ILC $v s$. 13.0\% with HER2 enriched in IDC, 5.9\% with TN in ILC vs. $12.0 \%$ with $\mathrm{TN}$ in IDC, $\mathrm{P}<0.001)$. Participants with ILC had smaller tumors than did those with IDC $(69.5 \%$ with T1 in ILC vs. $60.9 \%$ with T1 in IDC, $\mathrm{P}=0.004)$. No difference was observed in the type of surgery between participants with IDC and ILC $(\mathrm{P}=0.836)$.

\section{Factors influencing the number of resected SLNs}

The mean number of resected SLNs was $4.0 \pm 1.9$. No difference was observed in the number of resected SLNs 
Table 2 General participant characteristics

\begin{tabular}{|c|c|c|c|c|c|}
\hline \multirow{2}{*}{ Characteristic } & \multicolumn{2}{|c|}{ IDC $(n=6,650)$} & \multicolumn{2}{|c|}{ ILC (n=272) } & \multirow{2}{*}{$P$ value } \\
\hline & $\mathrm{N}$ & $\%$ & $\mathrm{~N}$ & $\%$ & \\
\hline Age at diagnosis* & & & & & 0.004 \\
\hline$\leq 40$ & 984 & 14.8 & 25 & 9.2 & \\
\hline $40-60$ & 4,180 & 62.9 & 168 & 61.8 & \\
\hline$>60$ & 1,486 & 22.3 & 79 & 29.0 & \\
\hline Molecular subtype & & & & & $<0.001$ \\
\hline Luminal A & 1,970 & 29.6 & 114 & 41.9 & \\
\hline Luminal B & 3,018 & 45.4 & 131 & 48.2 & \\
\hline HER2 enriched & 867 & 13.0 & 11 & 4.0 & \\
\hline TNBC & 795 & 12.0 & 16 & 5.9 & \\
\hline Pathologic tumor size & & & & & 0.004 \\
\hline T1 & 4,052 & 60.9 & 189 & 69.5 & \\
\hline $\mathrm{T} 2$ & 2,473 & 37.2 & 75 & 27.6 & \\
\hline T3-T4 & 125 & 1.9 & 8 & 2.9 & \\
\hline Surgical treatment & & & & & 0.836 \\
\hline $\begin{array}{l}\text { Breast-conserving } \\
\text { surgery }\end{array}$ & 2,044 & 30.7 & 82 & 30.1 & \\
\hline Mastectomy & 4,606 & 69.3 & 190 & 69.9 & \\
\hline
\end{tabular}

${ }^{*}$, the patients were divided into three groups according to age: $\leq 40$ (the young group), 40-60 (the middle age group) and $>60$ (the old group). IDC, invasive ductal carcinoma; ILC, invasive lobular carcinoma; HER2, human epidermal growth factor receptor 2; TNBC, triple-negative breast cancer.

between patients with IDC and ILC (IDC: $4.0 \pm 1.9 v s$. ILC: 3.9 $\pm 1.6, \mathrm{P}=0.352$ ). As shown in Table 3, age at diagnosis, tumor size, and tracer method were influencing factors of the number of resected SLNs in patients with IDC. For participants with ILC, tracer method was the only influencing factor of the number of resected SLNs. When a single mapping agent was used, the number of resected SLNs was more than that when mapping was performed with dualtracer in both participant groups (IDC: $4.1 \pm 1.9$ vs. 3.6 \pm 1.9 , $\mathrm{P}<0.001$, ILC: $4.0 \pm 1.6$ vs. $3.3 \pm 1.8, \mathrm{P}=0.034)$. In the subgroup analysis, there was no statistical difference in the number of resected SLNs between participants with IDC and ILC.

\section{SLNs and non-SLNs involvement in IDC and ILC}

In this study, SLNs or non-SLNs were considered positive for metastasis if they contained a macro- or micro-
Table 3 Association between clinicopathological factors and number of resected SLNs among invasive breast cancer patients

\begin{tabular}{|c|c|c|c|}
\hline \multirow{2}{*}{ Factors } & \multicolumn{2}{|c|}{ Number of resected SLNs $(\bar{x} \pm \mathrm{S})$} & \multirow{2}{*}{$P$ value } \\
\hline & IDC & ILC & \\
\hline \multicolumn{4}{|l|}{ Age at diagnosis } \\
\hline$\leq 40$ & $4.1 \pm 1.9$ & $4.0 \pm 1.5$ & 0.935 \\
\hline $40-60$ & $4.1 \pm 1.9$ & $3.8 \pm 1.6$ & 0.063 \\
\hline$>60$ & $3.8 \pm 1.9$ & $4.0 \pm 1.8$ & 0.335 \\
\hline$P$ value & $<0.001$ & 0.621 & \\
\hline \multicolumn{4}{|l|}{ Molecular subtype } \\
\hline Luminal A & $4.0 \pm 1.9$ & $3.8 \pm 1.6$ & 0.299 \\
\hline Luminal B & $4.0 \pm 1.9$ & $4.0 \pm 1.7$ & 0.785 \\
\hline HER2 enriched & $4.0 \pm 1.9$ & $3.6 \pm 1.2$ & 0.523 \\
\hline TNBC & $4.1 \pm 1.9$ & $3.5 \pm 1.8$ & 0.229 \\
\hline$P$ value & 0.803 & 0.480 & \\
\hline \multicolumn{4}{|l|}{ Pathologic tumor size } \\
\hline $\mathrm{T} 1$ & $3.9 \pm 1.9$ & $3.9 \pm 1.6$ & 0.619 \\
\hline T2 & $4.1 \pm 1.9$ & $4.0 \pm 1.8$ & 0.675 \\
\hline T3-T4 & $4.5 \pm 1.9$ & $3.6 \pm 1.7$ & 0.219 \\
\hline$P$ value & $<0.001$ & 0.654 & \\
\hline \multicolumn{4}{|l|}{ Tracer method } \\
\hline $\begin{array}{l}\text { A single mapping } \\
\text { agent }\end{array}$ & $4.1 \pm 1.9$ & $4.0 \pm 1.6$ & 0.659 \\
\hline Dual-tracer agent & $3.6 \pm 1.9$ & $3.3 \pm 1.8$ & 0.429 \\
\hline$P$ value & $<0.001$ & 0.034 & \\
\hline
\end{tabular}

SLNs, sentinel lymph nodes; IDC, invasive ductal carcinoma; ILC, invasive lobular carcinoma; HER2, human epidermal growth factor receptor 2; TNBC, triple-negative breast cancer.

metastasis. As shown in Table 4, the positive rate of SLNs was higher in participants with IDC than that in those with ILC $(19.3 \%$ in IDC vs. $12.9 \%$ in ILC, $\mathrm{P}=0.008)$. The difference in positive rate of SLNs between IDC and ILC was mainly contributed by macro-metastasis (macrometastasis rate: $17.1 \%$ in IDC $v s .11 .8 \%$ in ILC, $\mathrm{P}=0.022$; micro-metastasis rate: $2.6 \%$ in IDC vs. $1.1 \%$ in ILC, $\mathrm{P}=0.118)$. No difference was observed in rate of isolated tumor cells, SLNR, and rate of positive SLNs $\geq 3$ between participants with IDC and ILC. For patients with positive SLNs who underwent ALND, the metastatic rate of nonSLNs in group ILC was higher than that in group IDC, but the difference was not statistically significant $(50.0 \%$ 
Table 4 SLNs and non-SLNs involvement in IDC and ILC cases

\begin{tabular}{lccc}
\hline Variables & $\begin{array}{c}\text { IDC }(\mathrm{n}=6,650), \\
\%\end{array}$ & $\begin{array}{c}\text { ILC }(\mathrm{n}=272), \\
\%\end{array}$ & $\mathrm{P}$ value \\
\hline SLNs involvement & & 12.9 & 0.008 \\
$\quad$ Positive rate of SLNs & 19.3 & 11.8 & 0.022 \\
Macro-positive rate & 17.1 & 1.1 & 0.118 \\
Micro-positive rate & 2.6 & 0.4 & 1.000 \\
$\begin{array}{l}\text { Rate of isolated tumor } \\
\text { cells }\end{array}$ & 0.5 & 31.4 & 0.387 \\
$\begin{array}{l}\text { SLNR } \geq 50 \% \\
\text { Positive SLNs } \geq 3\end{array}$ & 39.0 & 14.3 & 0.796 \\
$\begin{array}{l}\text { Non-SLNs involvement } \\
\text { Metastatic rate of non- }\end{array}$ & 39.9 & 50.0 & 0.317 \\
SLNs & & & \\
$\begin{array}{l}\text { Metastatic rate of non- } \\
\text { SLNs in 1-2 positive } \\
\text { SLNs }\end{array}$ & 34.8 & 45.4 & 0.366 \\
\hline
\end{tabular}

*, non-SLNs metastases were calculated in patients with positive SLNs and received ALND. SLNs, sentinel lymph nodes; SLNR, sentinel lymph node ratio; IDC, invasive ductal carcinoma; ILC, invasive lobular carcinoma.

in ILC vs. $39.9 \%$ in IDC, $\mathrm{P}=0.317)$. For patients with 1-2 positive SLNs, metastatic rate of non-SLNs in the ILC group was also higher than that in the IDC group, but the difference was not statistically significant $(45.4 \%$ in ILC $v s$. $34.8 \%$ in IDC, $\mathrm{P}=0.366$ ). As shown in Table 5, for patients with positive SLNs who underwent ALND, no difference was observed between metastatic rate of non-SLNs and clinicopathological factors $(\mathrm{P}>0.05)$, including age at diagnosis, molecular subtype, pathologic tumor size, and tracer method.

\section{Influencing factors affecting positive rate of SLNs in IDC and ILC}

As shown in Table 6, in the subgroup analysis, the difference in positive rate of SLNs between IDC and ILC came from participants with luminal B and T1 (luminal B: $21.6 \%$ in IDC vs. $11.5 \%$ in ILC, $\mathrm{P}=0.005$; T1: $15.4 \%$ in IDC vs. $9.5 \%$ in ILC, $\mathrm{P}=0.028)$. Age at diagnosis, molecular subtype, and tumor size were factors influencing the positive rate of SLNs in patients with IDC. For patients with ILC, tumor size was the only factor influencing the positive rate of SLNs. To further confirm the factors affecting the
Table 5 Association between clinicopathological factors and metastatic rate of non-SLNs among invasive breast cancer patients*

\begin{tabular}{|c|c|c|}
\hline \multirow{2}{*}{ Factors } & \multicolumn{2}{|c|}{ Metastatic rate of non-SLNs } \\
\hline & IDC & ILC \\
\hline \multicolumn{3}{|l|}{ Age at diagnosis } \\
\hline$\leq 40$ & $46.4 \%$ & - \\
\hline $40-60$ & $39.4 \%$ & $52.4 \%$ \\
\hline$>60$ & $35.9 \%$ & $40.0 \%$ \\
\hline$P$ value & 0.132 & 1.000 \\
\hline \multicolumn{3}{|l|}{ Molecular subtype } \\
\hline Luminal A & $40.2 \%$ & $46.2 \%$ \\
\hline Luminal B & $41.0 \%$ & $50.0 \%$ \\
\hline HER2 enriched & $43.6 \%$ & $100.0 \%$ \\
\hline TNBC & $33.7 \%$ & - \\
\hline$P$ value & 0.504 & 1.000 \\
\hline \multicolumn{3}{|l|}{ Pathologic tumor size } \\
\hline T1 & $35.9 \%$ & $41.7 \%$ \\
\hline $\mathrm{T} 2$ & $43.5 \%$ & $53.8 \%$ \\
\hline T3-T4 & $42.9 \%$ & $100.0 \%$ \\
\hline$P$ value & 0.057 & 0.695 \\
\hline \multicolumn{3}{|l|}{ Tracer method } \\
\hline A single mapping agent & $40.8 \%$ & $56.5 \%$ \\
\hline Dual-tracer agent & $34.4 \%$ & $0.0 \%$ \\
\hline$P$ value & 0.203 & 0.458 \\
\hline
\end{tabular}

*, non-SLNs metastases were calculated in patients with positive SLNs and received ALND; -, no ILC cases were younger than 40 years old or were TN subtype. IDC, invasive ductal carcinoma; ILC, invasive lobular carcinoma; SLNs, sentinel lymph nodes; ALND, axillary lymph node dissection; TN, triple-negative.

positive rate of SLNs in patients with IDC, multivariable analyses were performed. As shown in Table 7, older age (age $>60, \mathrm{OR}=0.78,95 \% \mathrm{CI}$ : 0.62 to $0.97 \mathrm{P}=0.023$ ) was an independent negative factor for positive rate of SLNs. Taking $\mathrm{T} 1$ as contrast, $\mathrm{T} 2$ (OR $=1.98,95 \% \mathrm{CI}: 1.73$ to 2.26 , $\mathrm{P}<0.001)$ was a positive independent factor for the positive rate of SLNs. Compared to luminal A, luminal B (OR $=1.22,95 \% \mathrm{CI}: 1.05$ to $1.42, \mathrm{P}=0.012$ ) was a positive independent factor for the positive rate of SLNs, while HER2 (OR $=0.74,95 \%$ CI: 0.59 to $0.94, \mathrm{P}=0.013$ ) and TN $(\mathrm{OR}=0.78,95 \% \mathrm{CI}: 0.62$ to $0.99, \mathrm{P}=0.043)$ were negative independent factors. 
Table 6 Association between clinicopathological factors and positive rate of SLNs in IDC and ILC cases

\begin{tabular}{|c|c|c|c|}
\hline \multirow{2}{*}{ Variables } & \multicolumn{2}{|c|}{ Positive rate of SLNs, \% } & \multirow{2}{*}{$P$ value } \\
\hline & IDC & ILC & \\
\hline \multicolumn{4}{|l|}{ Age at diagnosis } \\
\hline$\leq 40$ & 21.3 & 8.0 & 0.106 \\
\hline $40-60$ & 19.6 & 15.5 & 0.186 \\
\hline$>60$ & 17.3 & 8.9 & 0.051 \\
\hline$P$ value & 0.035 & 0.309 & \\
\hline \multicolumn{4}{|l|}{ Molecular subtype } \\
\hline Luminal A & 18.2 & 14.9 & 0.378 \\
\hline Luminal B & 21.6 & 11.5 & 0.005 \\
\hline HER2 enriched & 13.5 & 9.1 & 1.000 \\
\hline TNBC & 16.2 & 6.3 & 0.491 \\
\hline$P$ value & $<0.001$ & 0.976 & \\
\hline \multicolumn{4}{|l|}{ Pathologic tumor size } \\
\hline $\mathrm{T} 1$ & 15.4 & 9.5 & 0.028 \\
\hline $\mathrm{T} 2$ & 25.8 & 21.3 & 0.387 \\
\hline T3-T4 & 20.0 & 12.5 & 1.000 \\
\hline$P$ value & $<0.001$ & 0.038 & \\
\hline \multicolumn{4}{|l|}{ Tracer method } \\
\hline A single mapping agent & 18.6 & 13.3 & 0.049 \\
\hline Dual-tracer agent & 21.0 & 6.3 & 0.043 \\
\hline $\mathrm{P}$ value & 0.091 & 0.390 & \\
\hline
\end{tabular}

SLNs, sentinel lymph nodes; IDC, invasive ductal carcinoma; ILC, invasive lobular carcinoma; HER2, human epidermal growth factor receptor 2; TNBC, triple-negative breast cancer.

\section{Discussion}

In the present nationwide cross-sectional study, we compared patients with ILC to those with IDC with respect to the implementation of SLNB and involvement of SLNs and non-SLNs. In this study, the characteristics of patients with ILC included older age at diagnosis, hormone receptor positivity and HER2 negativity, and smaller tumors; these observations were consistent with previous reports of ILC features (14). Moreover, no difference was observed in the number of resected SLNs between patients with IDC and ILC.

In the past, several studies have focused on the comparison of the risk of non-SLNs involvement
Table 7 Multivariate analyses for positive rate of SLNs among IDC patients

\begin{tabular}{lcc}
\hline Variables & OR $(95 \% \mathrm{Cl})$ & P value \\
\hline Age at diagnosis & 1.00 & \\
$\leq 40$ & $0.92(0.76-1.10)$ & 0.350 \\
$40-60$ & $0.78(0.62-0.97)$ & 0.023 \\
$>60$ & & \\
Molecular subtype & 1.00 & \\
Luminal A & $1.22(1.05-1.42)$ & 0.012 \\
Luminal B & $0.74(0.59-0.94)$ & 0.013 \\
HER2 enriched & $0.78(0.62-0.99)$ & 0.043 \\
TNBC & 1.00 & \\
Pathologic tumor size & $1.98(1.73-2.26)$ & $<0.001$ \\
T1 & $1.45(0.91-2.31)$ & 0.118 \\
T2 & & 0.065 \\
T3-T4 & 1.00 & \\
Tracer method & $0.99-1.41)$ & \\
A single mapping agent & & \\
Dual-tracer agent & & \\
\hline
\end{tabular}

SLNs, sentinel lymph nodes; IDC, invasive ductal carcinoma; ILC, invasive lobular carcinoma; HER2, human epidermal growth factor receptor 2; TNBC, triple-negative breast cancer; OR, odds ratio; $\mathrm{Cl}$, confidence interval.

between patients with IDC and those with ILC. However, information regarding the implementation of SLNB in patients with ILC is sparse. Our study showed that the positive rate of SLNs was higher in participants with IDC than that in those with ILC (19.3\% in IDC $v s .12 .9 \%$ in ILC, $\mathrm{P}=0.008)$. The difference in positive rate of SLNs between IDC and ILC was mainly attributed to macrometastasis. This finding is similar to previous studies that reported that $18 \%$ ILC and $21 \%$ IDC cases were SLN micro-metastasis and macro-metastasis in 171 ILC and 2,168 IDC cases (11). There was no significant difference in the positive rate of SLNs between the ILC and IDC groups in the previous research $(\mathrm{P}=0.36)$, which may be due to the race/ethnicity difference of patients and the number of cases. In addition, several studies have found that ILC tended to have higher numbers of positive nodes when it metastasized to ALNs (19,20); however, few studies have explored the difference in the number of positive SLNs between ILC cases and IDC cases. In our study, no 
difference was observed in SLNR and rate of positive SLNs $\geq 3$ between patients with IDC and ILC, which indicates that the number of positive SLNs seems to be no different between patients with ILC and IDC.

Several studies have shown that different histological types of breast cancer incur different ALN metastasis burdens. Local removal of SLNs in early breast cancer will change the treatment and surgical strategies of breast cancer. Based on existing clinical trial evidence, if radiotherapy and adequate adjuvant systemic treatments are planned, the omission of ALND and SLNB with 1-2 positive SLNs following breast conserving surgery is not harmful (8). In addition, ALND is not justified for patients with micro-positive sentinel node involvement and that this does not impact on survival. However, for patients with micro-positive sentinel lymph nodes after neoadjuvant chemotherapy, ALND is still recommended for these patients (21). Whether ILC patients with 1-2 positive SLNs can avoid ALND remains controversial.

Many studies have shown that ILC was associated with a high risk of non-SLN involvement or ILC had more non-SLN metastasis than IDC. For example, Majid et al. found that ILC was associated with a high risk of nonSLN involvement (OR $=1.73,95 \%$ CI: 1.01 to 2.97) (22). Adachi et al. discovered that ILC cases had more non-SLN metastasis than IDC cases among SLN macro-metastasis patients $(68 \%$ in ILC $v s .46 \%$ in IDC, $\mathrm{P}=0.03)(11)$. Fernández et al. found that ILC was associated with a higher ratio of positive lymph nodes $(0.46 \pm 0.30$ in ILC and $0.33 \pm 0.23$ in IDC, $\mathrm{P}<0.01)(19)$. In our study, for participants with positive SLNs who received ALND, the positive rate of non-SLNs in the ILC group was higher than that in the IDC group (50.0\% in ILC vs. $39.9 \%$ in IDC), but the difference was not statistically significant. However, a few studies have shown inconsistent conclusions. Corona et al. discovered that ILC histology is not associated with the risk of further metastasis at ALND (OR $=1.62,95 \%$ CI: 0.77 to $3.41, \mathrm{P}=0.20$ ) (13). Gao et al. found that ILC had similar rates of non-SLN metastasis compared with IDC among patients with $1-2$ positive SLNs (31.2\% in ILC vs. $28.6 \%$ in IDC, $\mathrm{P}=0.481)(12)$; however, in their study, there were only 182 IDC and 5 ILC patients with 1-2 positive SLNs (12). In our study, the metastatic rate of non-SLNs in group ILC was $10.4 \%$ higher than that in group IDC among patients with 1-2 positive SLNs, although the difference was not statistically significant (45.4\% in ILC, $\mathrm{n}=30$ vs. $34.8 \%$ in IDC, $\mathrm{n}=1,122, \mathrm{P}=0.366$ ). Therefore, surgeons should be more cautious about omitting ALND for ILC patients with 1-2 positive SLNs.

The predictors for the involvement of lymph nodes have been widely studied, while the risk factors for positive SLNs have rarely been explored. We found that tumor size was the only influencing factor affecting positive rate of SLNs in participants with ILC, which was in line with a previous study (23). For patients with IDC, age at diagnosis, molecular subtype, and pathologic tumor size were independent factors for the positive rate of SLNs. Falco et al. found that large primary tumor diameter $(\mathrm{P}=0.0132)$, molecular type $(\mathrm{P}=0.0492)$ and amount of positive SLNs $(\mathrm{P}=0.0408)$ were risk factors for positive ALNs based on a total of 391 patients with positive SLNs (24). Chakraborty et al. analyzed 426 patients with breast cancer, and they found that age, tumor grade, and tumor size were likely to be associated with number of lymph node metastasis (25). Among 814 patients with T1 and T2 primary breast cancer, Si et al. found that luminal HER2 - and luminal HER2+ type showed a significantly higher probability of lymph nodes involvement when using triple negative breast cancer (TNBC) as a reference (26). The relationship between SLNs metastasis and age at diagnosis and $\mathrm{T}$ stage seems to be similar to that of theirs and lymph nodes, but further study is needed about relationship between molecular subtype and SLNs metastasis.

Our database included patients from 37 medical centers in China, who were treated in different settings. The range of participants was broad: teenagers to elderly people were included. Therefore, our results could be applicable to other IDC and ILC patients. The present study had some potential limitations. First, the number of participants with ILC was relatively low compared to those with IDC, which might be due to the low prevalence of ILC. Second, there was no patient follow-up conducted in this study, so the prognosis of patients with IDC and ILC could not be compared. Therefore, further studies with a larger cohort and follow-up are needed in patients with IDC and ILC.

\section{Conclusions}

This study, including a nationwide multi-center cohort of patients with IDC and ILC, demonstrated that patients with ILC had a similar number of resected SLNs, lower positive rate of SLNs, and increased tendency for non-SLNs metastasis compared to those with IDC. In patients with 1-2 positive SLNs, the ILC group also had an increased tendency for non-SLNs metastasis compared with the IDC group. Therefore, different histological types of invasive breast 
cancer lead to different SLNs and non-SLNs metastasis burdens. Surgeons may need to be more cautious about omitting ALND for ILC patients with 1-2 positive SLNs.

\section{Acknowledgments}

The authors extend their thanks to the following members of Chinese Society of Breast Surgery for providing data: Hongchuan Jiang, Beijing Chaoyang Hospital; Xuening Duan, Peking University First Hospital; Shu Wang, Peking University People's Hospital; Hua Kang, Xuanwu Hospital of Capital Medical University; Xiang Qu, Beijing Friendship Hospital; Zuowei Zhao, The Second Hospital of Dalian Medical University; Chuan Wang, Fujian Medical University Union Hospital; Kejin Wu, The Obstetrics \& Gynecology Hospital of Fudan University; Qiang Zou, Huashan Hospital of Fudan University; Wei Zhu, Zhongshan Hospital of Fudan University; Haiyun Huang, Gansu Provincial Hospital; Suisheng Yang, Gansu Provincial Cancer Hospital; Dahua Mao, Affiliated Wudang Hospital of Guizhou Medical University; Jianguo Zhang, The Second Affiliated Hospital of Harbin Medical University; Yunjiang Liu, The Fourth Hospital of Hebei Medical University; Zhenzhen Liu, Henan Cancer Hospital; Ke Liu, Jilin Cancer Hospital; Shui Wang, Jiangsu Province Hospital; Jiandong Wang, The General Hospital of the People's Liberation Army; Li Wu, The First Hospital of Lanzhou University; Ailin Song, The Second Hospital of Lanzhou University; Jun Jiang, Yi Zhang, Southwest Hospital; Yonghui Luo, The second affiliated Hospital of Nanchang University; Zhongwei Cao, Inner Mongolia Autonomous Region People's Hospital; Zhigang Yu, Second Hospital of Shandong University; Xingsong Tian, Shandong Provincial Western Hospital; Jinping Liu, Sichuan Provincial People's Hospital; Jianghua Ou, Affiliated Cancer Hospital of Xinjiang Medical University; Dedian Chen, Yunnan Cancer Hospital; Peifen $\mathrm{Fu}$, The First Affiliated Hospital of Zhejiang University; Jian Huang, The Second Affiliated Hospital of Zhejiang University; Feng Jin, The First Hospital of China Medical University; Yi Zhao, Shengjing Hospital of China Medical University; Lili Tang, Xiangya Hospital Central South University; and Erwei Song, Sun Yat-sen Memorial Hospital of Sun Yat-sen University.

Funding: This paper was funded by Key R\&D Program of Shaanxi Province, China (2018ZDXM-SF-066) and Key Project of Natural Science Foundation of Shaanxi Province, China (2021JZ-29).

\section{Footnote}

Reporting Checklist: The authors have completed the STROBE reporting checklist. Available at https://dx.doi. org/10.21037/atm-21-5169

Data Sharing Statement: Available at https://dx.doi. org/10.21037/atm-21-5169

Conflicts of Interest: All authors have completed the ICMJE uniform disclosure form (available at https://dx.doi. org/10.21037/atm-21-5169). The authors have no conflicts of interest to declare.

Etbical Statement: The authors are accountable for all aspects of the work in ensuring that questions related to the accuracy or integrity of any part of the work are appropriately investigated and resolved. This study conformed to the provisions of the Declaration of Helsinki (as revised in 2013) and was approved by the Ethical Committee of Xijing Hospital, The Fourth Military Medical University (KY20192114-C-1). Individual consent for this retrospective analysis was waived.

Open Access Statement: This is an Open Access article distributed in accordance with the Creative Commons Attribution-NonCommercial-NoDerivs 4.0 International License (CC BY-NC-ND 4.0), which permits the noncommercial replication and distribution of the article with the strict proviso that no changes or edits are made and the original work is properly cited (including links to both the formal publication through the relevant DOI and the license). See: https://creativecommons.org/licenses/by-nc-nd/4.0/.

\section{References}

1. Tyagi NK, Dhesy-Thind S. Clinical practice guidelines in breast cancer. Curr Oncol 2018;25:S151-60.

2. Dixon JM, Cartlidge CWJ. Twenty-five years of change in the management of the axilla in breast cancer. Breast J 2020;26:22-6.

3. Veronesi U, Paganelli G, Viale G, et al. A randomized comparison of sentinel-node biopsy with routine axillary dissection in breast cancer. N Engl J Med 2003;349:546-53.

4. Callejo IP, Brito JA, Bivar JW, et al. Predictors of positive axillary lymph nodes in breast cancer patients with metastatic sentinel lymph node. Clin Transl Oncol 2005;7:18-22.

5. Viale G, Zurrida S, Maiorano E, et al. Predicting the status 
of axillary sentinel lymph nodes in 4351 patients with invasive breast carcinoma treated in a single institution. Cancer 2005;103:492-500.

6. Patani NR, Dwek MV, Douek M. Predictors of axillary lymph node metastasis in breast cancer: a systematic review. Eur J Surg Oncol 2007;33:409-19.

7. Charalampoudis P, Markopoulos C, Kovacs T. Controversies and recommendations regarding sentinel lymph node biopsy in primary breast cancer: A comprehensive review of current data. Eur J Surg Oncol 2018;44:5-14.

8. Magnoni F, Galimberti V, Corso G, et al. Axillary surgery in breast cancer: An updated historical perspective. Semin Oncol 2020;47:341-52.

9. Giuliano AE, Hunt KK, Ballman KV, et al. Axillary dissection vs no axillary dissection in women with invasive breast cancer and sentinel node metastasis: a randomized clinical trial. JAMA 2011;305:569-75.

10. Giuliano AE, Ballman KV, McCall L, et al. Effect of Axillary Dissection vs No Axillary Dissection on 10-Year Overall Survival Among Women With Invasive Breast Cancer and Sentinel Node Metastasis: The ACOSOG Z0011 (Alliance) Randomized Clinical Trial. JAMA 2017;318:918-26.

11. Adachi Y, Sawaki M, Hattori M, et al. Comparison of sentinel lymph node biopsy between invasive lobular carcinoma and invasive ductal carcinoma. Breast Cancer 2018;25:560-5.

12. Gao W, Zeng Y, Fei X, et al. Axillary lymph node and non-sentinel lymph node metastasis among the ACOSOG Z0011 eligible breast cancer patients with invasive ductal, invasive lobular, or other histological special types: a multi-institutional retrospective analysis. Breast Cancer Res Treat 2020;184:193-202.

13. Corona SP, Bortul M, Scomersi S, et al. Management of the axilla in breast cancer: outcome analysis in a series of ductal versus lobular invasive cancers. Breast Cancer Res Treat 2020;180:735-45.

14. Thomas M, Kelly ED, Abraham J, et al. Invasive lobular breast cancer: A review of pathogenesis, diagnosis, management, and future directions of early stage disease. Semin Oncol 2019;46:121-32.

15. McCart Reed AE, Kutasovic JR, Lakhani SR, et al. Invasive lobular carcinoma of the breast: morphology, biomarkers and 'omics. Breast Cancer Res 2015;17:12.

16. Lopez JK, Bassett LW. Invasive lobular carcinoma of the breast: spectrum of mammographic, US, and MR imaging findings. Radiographics 2009;29:165-76.

17. Cristofanilli M, Gonzalez-Angulo A, Sneige N, et al. Invasive lobular carcinoma classic type: response to primary chemotherapy and survival outcomes. J Clin Oncol 2005;23:41-8.

18. Zhang J, Wang T, Yan C, et al. Clinical Practice Status of Sentinel Lymph Node Biopsy for Early-Stage Breast Cancer Patients in China: A Multicenter Study. Clin Epidemiol 2020;12:917-24.

19. Fernández B, Paish EC, Green AR, et al. Lymph-node metastases in invasive lobular carcinoma are different from those in ductal carcinoma of the breast. J Clin Pathol 2011;64:995-1000

20. Topps A, Clay V, Absar M, et al. The sensitivity of preoperative axillary staging in breast cancer: comparison of invasive lobular and ductal carcinoma. Eur J Surg Oncol 2014;40:813-7.

21. Burstein HJ, Curigliano G, Loibl S, et al. Estimating the benefits of therapy for early-stage breast cancer: the St. Gallen International Consensus Guidelines for the primary therapy of early breast cancer 2019. Ann Oncol 2019;30:1541-57.

22. Majid S, Rydén L, Manjer J. Determinants for nonsentinel node metastases in primary invasive breast cancer: a population-based cohort study of 602 consecutive patients with sentinel node metastases. BMC Cancer 2019;19:626.

23. Güven HE, Kültüroğlu MO, Gülçelik MA, et al. Sentinel Lymph Node Metastasis in Invasive Lobular Carcinoma of the Breast. Eur J Breast Health 2018;14:117-20.

24. Falco M, Masojć B, Byrski T, et al. The diameter of metastasis in positive sentinel lymph node biopsy affects axillary tumor load in early breast cancer. Asia Pac J Clin Oncol 2019;15:121-7.

25. Chakraborty A, Bose CK, Basak J, et al. Determinants of lymph node status in women with breast cancer: A hospital based study from eastern India. Indian J Med Res 2016;143:S45-51.

26. Si C, Jin Y, Wang H, et al. Association between molecular subtypes and lymph node status in invasive breast cancer. Int J Clin Exp Pathol 2014;7:6800-6.

(English Language Editor: J. Jones)

Cite this article as: Zhang J, Ling Y, Wang T, Yan C, Huang M, Fan Z, Ling R; Chinese Society of Breast Surgery. Analysis of sentinel lymph node biopsy and non-sentinel lymph node metastasis in invasive ductal and invasive lobular breast cancer: a nationwide cross-sectional study (CSBrS-001). Ann Transl Med 2021;9(20):1588. doi: 10.21037/atm-21-5169 